\title{
Water Hazard Characteristics of Dabaoshan Atypical Karst Tunnel
}

\author{
Liu Shao-Ming ${ }^{1}$, Chen An ${ }^{2,}$, Liu Hao ${ }^{1}$, Luo Sha-Sha ${ }^{2}$ \\ ${ }^{1}$ Guangdong Transportation Technology Testing Co, Ltd, Guangzhou, China \\ ${ }^{2}$ Faculty of Land Resource Engineering, Kunming University of Science and Technology, Kunming, China \\ Email address: \\ 214872665@qq.com (Liu Shao-Ming), csuchenan@126.com(Chen An),308652550@qq.com (Liu Hao), \\ 1124295846@qq.com (Luo Sha-Sha) \\ *Corresponding author
}

To cite this article:

Liu Shao-Ming, Chen An, Liu Hao, Luo Sha-Sha. Water Hazard Characteristics of Dabaoshan Atypical Karst Tunnel. American Journal of Civil Engineering. Vol. 7, No. 2, 2019, pp. 59-63. doi: 10.11648/j.ajce.20190702.14

Received: April 22, 2019; Accepted: May 22, 2019; Published: June 15, 2019

\begin{abstract}
To study the water hazard characteristics of DaBaoshan atypical karst tunnel. Engineering geological and hydrogeological mapping and survey, water connection test, tunnel water hazard investigation, theoretical calculation and analysis are used. The results show that the lithology of the DaBaoshan tunnel is argillaceous limestone, limestone, carbonaceous shale limestone, limestone and inferior coal seam, which is an atypical karst tunnel. There are three karst collapses, but there are not perennial water bodies on the surface of tunnel hill. In the rainy season, there are gushing water and mud in the tunnel, the water source of tunnel water hazard is rainfall, and the gushing water channels are surface collapse, dissolved crack and fissure. The calculation results show the rainfall intensity are $7.84 \mathrm{~mm} / \mathrm{h}, 8.44 \mathrm{~mm} / \mathrm{h}, 9.18 \mathrm{~mm} / \mathrm{h}, 10.17 \mathrm{~mm} / \mathrm{h}$ respectively, the rainfall time that lead to the gushing water exceeds the drainage capacity of the gutters are $2.5 \mathrm{~h}, 2.2 \mathrm{~h}, 2.0 \mathrm{~h}, 1.8 \mathrm{~h}$ respectively. And when the rainfall intensity is $33.3 \mathrm{~mm} / \mathrm{h}$, the rainfall time is only $35 \mathrm{~min}$. The tunnel gushing water causes a lot of water, mud and sands flows on the road which endangers traffic safety, even causing traffic accidents.
\end{abstract}

Keywords: Water Hazard Characteristic, Dabaoshan Tunnel, Atypical Karst

\section{Introduction}

Water hazard is one of the five major disasters that threaten the safety of tunnels. As early as 1986, Wei Sen studied the cause, the harm and the prevention technology of water hazard during karst tunnel construction [1]. In 2000, Wan De-you expounded the comprehensive treatment measures for water hazard in operational railway karst tunnels [2]. In 2001, $\mathrm{Li}$ Huai-jian analyzed the reasons and introduced the treatment technology for the leakage of one tunnel in the BoLai expressway [3]. In 2003, Zhu He-quan and Li Haiyan summarized the main waterproof technology for railway tunnels in China [4]. In 2005, on the basis of analyzing the water hazard, He Zhong-ming used surface drilling and grouting method to treat water hazard [5]. In 2007, Li Xian-wei investigated the intrinsic relationship between groundwater hazard and related geological disasters of QinLing karst tunnel [6]. In 2008, Xue Yu-guo and Li Shu-cai introduced the methods of water hazard detection during high-risk karst tunnel construction [7]. In the same year, Xu Changchun proposed a karst tunnel water hazard treatment technology based on "chain theory" [8]. In 2010, Ma Dong summarize the solutions for complex karst tunnels to avoid risks, controll water disasters base on the DaZhiPing karst tunnel [9]. In 2011, Zhang Min discussed the treatment of water leakage in karst tunnels [10]. In 2012, Huang Ke used the directional grouting technology to control the water hazard in Zhu Zi-lin station karst tunnel in Shenzhen [11]. Liu Jian and Liu Dan used SVM technology to establish the source identification model of water inrush in karst tunnels [12]. In 2013, Shi Ming discussed the water inrush phenomenon and the groundwater harm treatment of the PingDong karst tunnel [13]. In 2014, Zhang Yan-long and Li Qing discussed the water hazard and treatment techniques of operating road karst tunnel [14]. Chen An studied the water hazard characteristics of PiShuangAo karst tunnel [15]. In 2015, Liu Li and Liu Qun evaluated the risk of water damage in karst tunnels by using 
analytic hierarchy process and fuzzy comprehensive evaluation theory relied on the ChangAnZhai karst tunnel, and formulated karst water hazard prevention and control measures according to the risk level [16]. In 2016, Zhang Yan-long and Li Qing studied the water hazard mechanism and evaluation technology of the EBuLing karst tunnel [17]. In 2017, Li Kai and Lin Hai-shan studied the water hazard mechanism of BaiShan tunnel [18]. Zhang Yan-long and Liu Hao conducted an assessment of the water damage of the Cha LinDing karst tunnel [19]. In 2018, Zhang Yan-long, Tian Qing-yan analyzed the water damage of highway karst tunnel and carried out numerical simulation analysis [20].

The above research mainly focuses on the study of water hazard during tunnel construction, or mainly for the evaluation and treatment of water hazard in karst tunnels. There are few studies on water hazard in operating atypical karst tunnel. In this paper, the Dabaoshan atypical karst tunnel is located in argillaceous limestone, limestone, carbonaceous limestone, carbonaceous shale, limestone and inferior coal seams, which is is one of the Beijing-Hong Kong-Macau expressway tunnels. The research results of Dabaoshan atypical karst tunnel can provide basic information for tunnel water damage control and provide reference for similar projects.

\section{Geological Background}
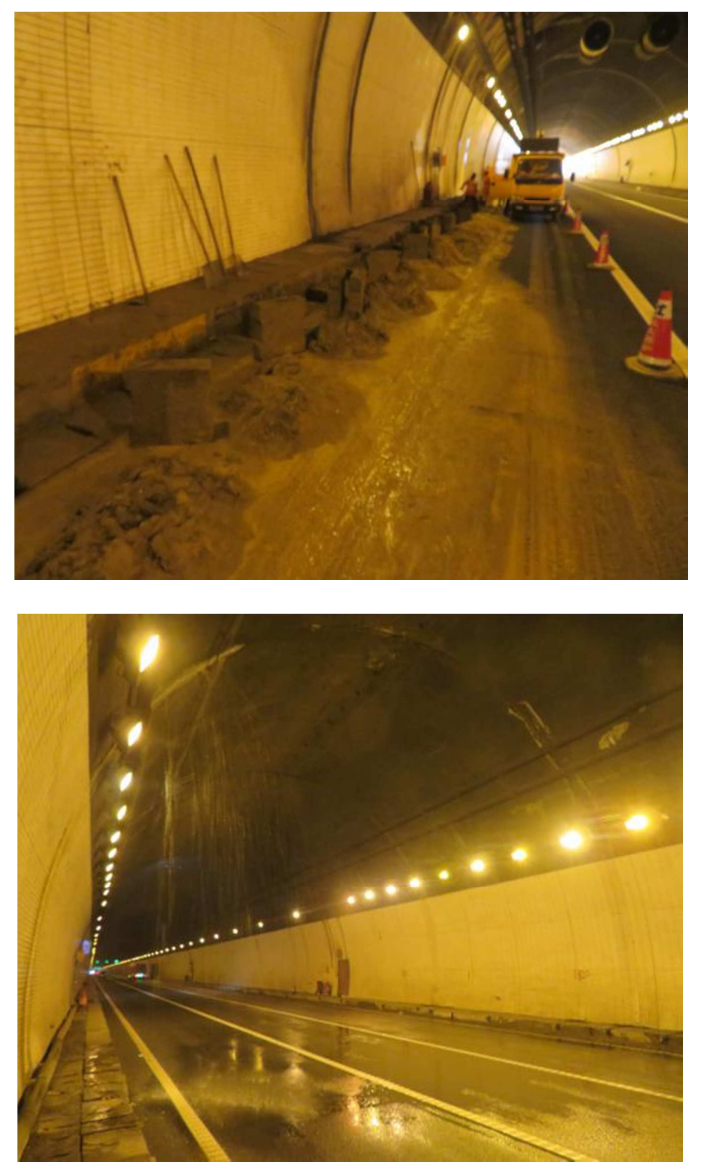

Figure 1. Gushing water and gushing mud in karst tunnel.

Dabaoshan atypical karst tunnel of Beijing-Hong
Kong-Macau expressway is located on the boundary of Qujiang and Wengyuan County, Shaoguan County, Guangdong Province, China. The entrance mileage of northbound line (left line) is NK1983+533, and the exit mileage is NK1985+118, which length is $1585 \mathrm{~m}$. The entrance mileage of southbound line (right line) is SK1983+553, and the exit mileage is SK1985+118, which length is $1565 \mathrm{~m}$. On May 10, 2018, the torrential rain falled in Shaoguan, and there was gushing water and mud at the mileage of NK1984+840 in Dabaoshan atypical karst tunnel. Serious water leakage occurred in other areas, which directly threatened the traffic safety (Figure 1).

Dabaoshan tunnel locates on hilly area, which has steep terrain and developed gully. The average annual rainfall in the tunnel area is $1640 \mathrm{~mm}$, but the rainy season accounts for $77.5 \%$ of the annual rainfall.

The tunnel traverses two anticlines and one fault. The first anticline is located in NK1983+450-NK1984+330, and the anticline axis is located at NK1984+090, which lithology is argillaceous limestone and limestone. The joint fissures are more developed and small karst caves developed. The fault is located at NK1984+370, which is a normal fault. The lower wall of the fault is argillaceous limestone and limestone of Devonian and the the upper wall of the fault is carbonaceous shale limestone, limestone and inferior coal seam of Jurassic. The fault dips to the south, the dip angle is $70^{\circ}-80^{\circ}$, which affects the tunnel length of about 60 meters. The second anticline is located in NK1984+330-NK1985+135, and the anticline axis is located at NK1984+705, which lithology is carbonaceous shale limestone, limestone and inferior coal seam. The joint fissures develop well and the rock mass is broken. The carbonaceous shale limestone, limestone and inferior coal seam is located in NK1984+370- NK1984+600, the limestone is located in NK1984+600- NK1985+130.

Within the range of NK1983+450-NK1984+330, the tunnel mountain strata are composed of medium-thick layered argillaceous limestone and limestone of Devonian, which is monoclinic structure with dip angle of $25^{\circ}-52^{\circ}$. The fissure developed well. Within the range of NK1984+330-NK1984+650, the tunnel mountain strata are composed of thin layered carbonaceous shale limestone, limestone and inferior coal seam of Jurassic, which is monoclinic structure with dip angle of $41^{\circ}-59^{\circ}$. The fissure developed well. Within the range of NK1984+650-NK1985+090, the tunnel mountain strata are composed of thin-medium layered carbonaceous shale limestone, limestone and inferior coal seam of Devonian, which is monoclinic structure with dip angle of $41^{\circ}-59^{\circ}$. The fissure developed well and the strata are deformed severely by extrusion.

\section{Karst Collapses}

Limestone karst of Dabaoshan atypical karst tunnel develops poor. There are no karst grooves, isolated peak, stone forest, karst funnels, sinkholes, clint and stalagmites on the surface of hill. 
There are three karst collapses on the mountain surface.

\subsection{Karst Collapse 1 (TX1)}

It is located at NK1984+836 above the tunnel (about $282 \mathrm{~m}$ from the exit), which near the exit of Dabaoshan tunnel of 106 National Road. It's shape is nearly rectangular. The length is $7.7 \mathrm{~m}$, the breadth is $6.5 \mathrm{~m}$, and the height is $2.5 \mathrm{~m}$ (Figure 2).

\subsection{Karst Collapse 2 (TX2)}

It is the old karst collapse, which surface has carried out hardening treatment. It is located at NK1983+933 above the tunnel (about $400 \mathrm{~m}$ from the entrance). It's shape is nearly rectangular. The length is $5.5 \mathrm{~m}$, the breadth is $2.5 \mathrm{~m}$, and the height is $0.5-0.8 \mathrm{~m}$ (Figure 3 ).

\subsection{Karst Collapse 3 (TX3)}

It is located at NK1983+610 above the tunnel. There are three collapses in this section (Figure 4).

The shape of collapse 3-1 (TX3-1) is nearly circular. Its radius is $35.0-40.0 \mathrm{~m}$ and its depth is about $15 \mathrm{~m}$. The shape of collapse 3-2 (TX3-2) is nearly oval, its long axis is $30 \mathrm{~m}$ and short axis is $20 \mathrm{~m}$, its depth is about $15 \mathrm{~m}$. The shape of collapse 3-3 (TX3-3) is nearly oval, its long axis is $20 \mathrm{~m}$ and short axis is $15 \mathrm{~m}$, its depth is about $5 \mathrm{~m}$.

It is speculated that there are caves, solution fissure and fissure under the karst collapses.

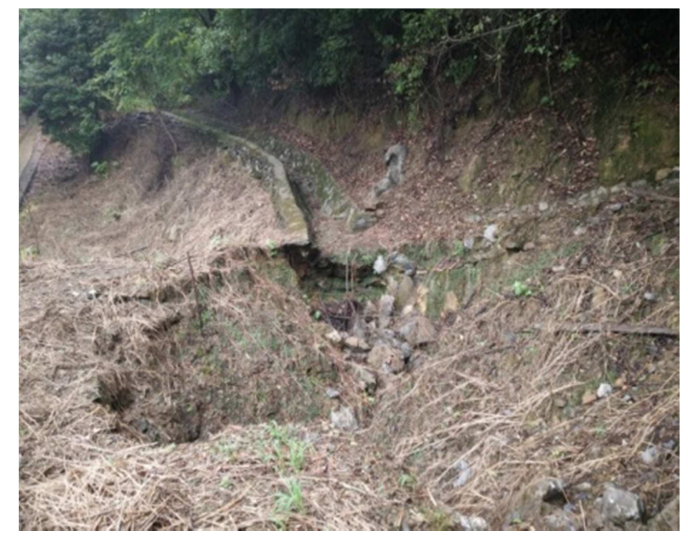

Figure 2. Karst collapse 1 (TX1).

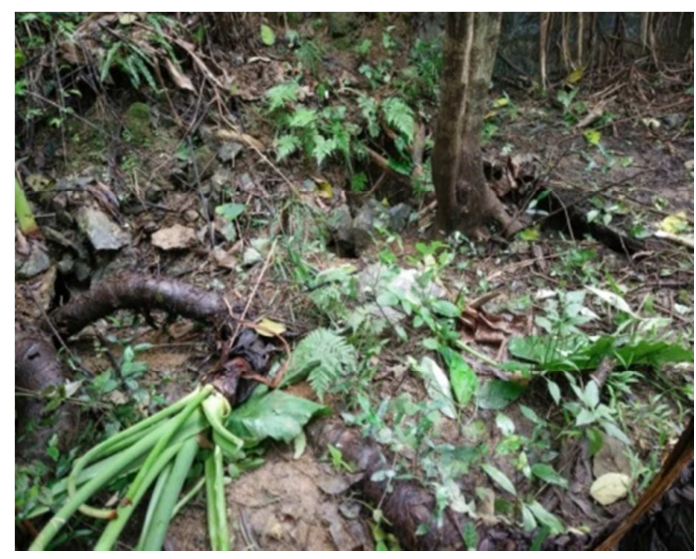

Figure 3. Karst collapse 2 (TX2).

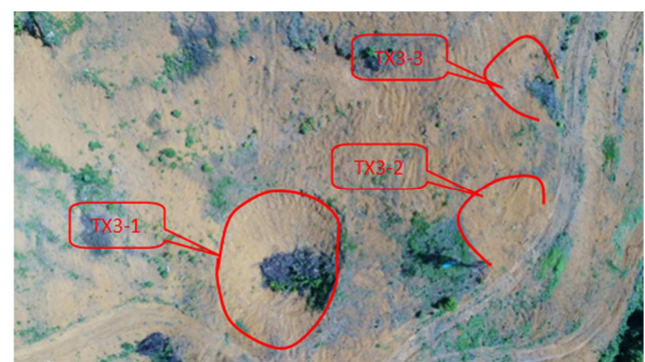

Figure 4. Karst collapse 3 on the surface of hill (TX3).

\section{Hydrogeological Conditions}

The river systems are not developed on the tunnel hill. There is not perennial surface water, and there is only a seasonal flood in the gully.

(1) In the mileage of NK1983+450-NK1984+330, the lithology is argillaceous limestone and limestone, and the small cave develops well in the shallow area. The groundwater stored in fissure and solution fissure, which water content is poor and water volume is small. Its main recharge source is rainfall.

(2) In the mileage of NK1984+330-NK1984+650, the lithology is carbonaceous shale limestone, limestone and inferior coal seam. The groundwater stored in fissure, which water content is poor and water volume is small. Its main recharge source is rainfall.

(3) In the mileage of NK1984+650-NK1985+090, the lithology is carbonaceous shale limestone, limestone and inferior coal seam, and the small cave develops well in the shallow area. The groundwater stored in fissure and solution fissure, which water content is poor and water volume is small. Its main recharge source is rainfall.

The groundwater is dominated by fissure water. Some strata is dissolved so there are karst solution fissures water.

The reagent dispensing points of groundwater connection test are karst collapsed 1, karst collapsed 2, and karst collapsed 3-1, respectively. The corresponding reagents were: rhodamine, fluorescent brightener, and fluorescein sodium.

The groundwater receiving points: (1) left line slow lane exit drain, (2) left line passing lane exit drain. The results of groundwater connection test show that the karst collapse on the surface of the hill is connected to the tunnel (Figure 5).

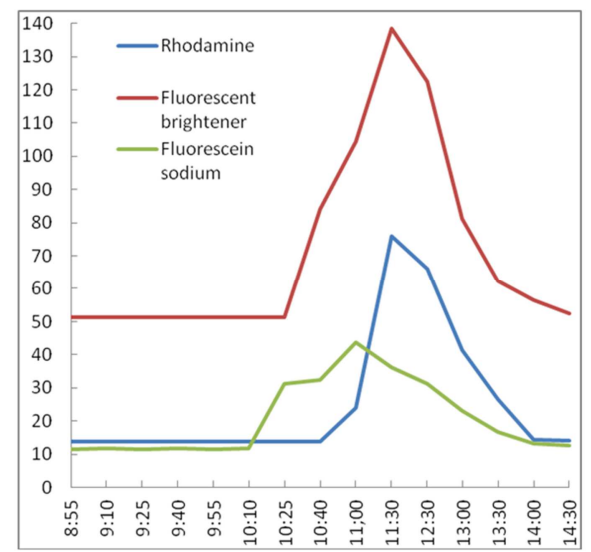




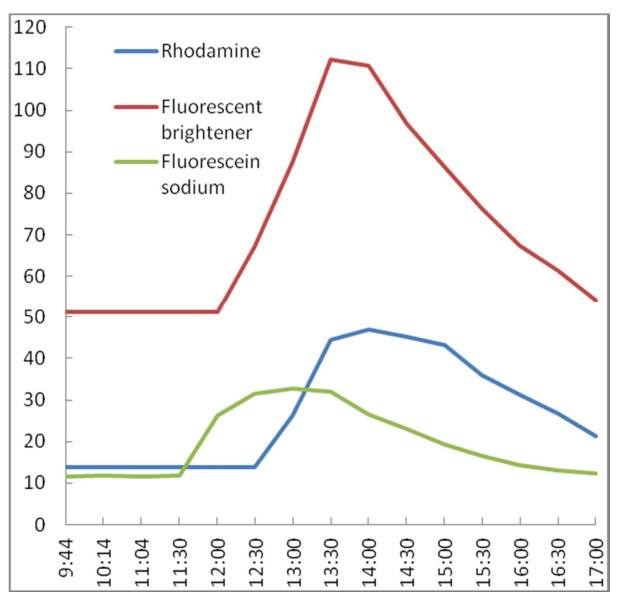

Figure 5. Groundwater connection test results (June 21, 2018).

\section{Water Hazard}

During tunnel construction, there were karst caves at NK1983+710, water and mud pouring into the tunnel. In the mileage of NK1984+260- NK1984+300, which exposes karst caves and the tunnel surrounding rockmass collapsed to the surface.

In May 2013, the heavy rains fell in Shaoguan area. The groundwater level increased rapidly, and the groundwater pressure on the tunnel lining increased sharply, which caused the emergence of the second lining concrete of the mileage of NK1983+978 in Dabaoshan tunnel (Figure 6).
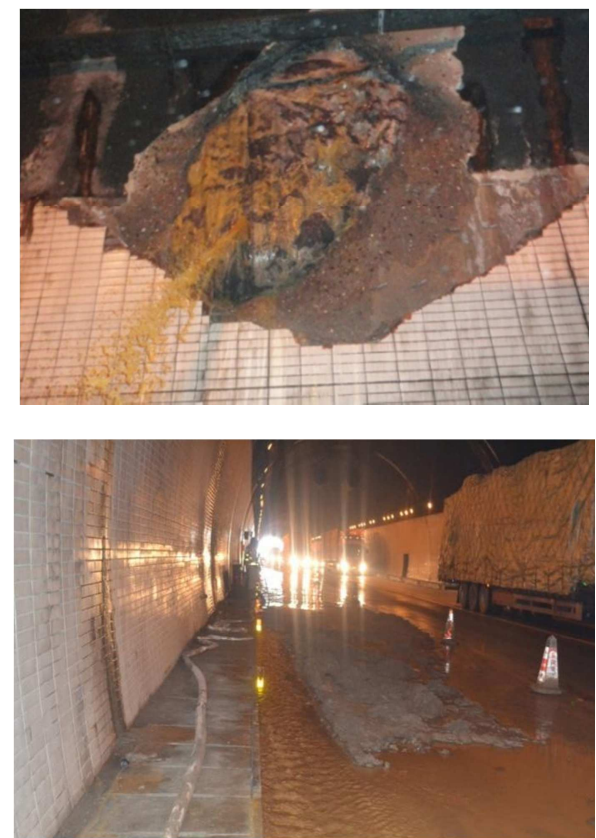

Figure 6. Lining off and gushing water in tunnel.

In May 2018, heavy rain fell in Shaoguan area, the groundwater level rose rapidly. As a result, there was gushing water and mud at the mileage of NK1984+840 in Dabaoshan tunnel, and severe water seepage and water leakage occurred in other areas (Figure 1), which seriously threatened the traffic safety.

\section{Water Hazard Characteristics}

\subsection{Gushing Water Sources}

There are not perennial water bodies on the surface or the periphery of the hill. There is not water drenching or water dripping substantially during dry season but the water pouring and water inrush is violent during rainy season. The groundwater connection test showed that surface karst collapse was connected to the tunnel. So the gushing water source of Dabaoshan tunnel is rainfall.

\subsection{Gushing Water Channels}

Limestone karst developed well. There are karst collapses on hill surface (figure 2, figure 3, figure 4 ). The three connectivity test results show that their concentration curves are very similar. They are single peak curves (figure 5). The times of peak values are two hours and it is indicate that rainfall smoothly flow into the tunnel. So the gushing water channels are karst collapses, karst caves, solution fissure and fissure.

\subsection{Water Hazard Time-pRocess Calculation}

The gushing water source of Dabaoshan tunnel is rainfall so the maximum gushing water is closely relate to rainfall intensity and rainfall time. The following formula can be used to calculate the maximum gushing water according to the daily maximum precipitation and precipitation time.

$$
\mathrm{Q}=\alpha \mathrm{AXT} \eta
$$

$\mathrm{Q}$ - the maximum gushing water, $\mathrm{m}^{3} / \mathrm{h}$

$\alpha$ - coefficient of precipitation infiltration, since the surface atmospheric precipitation of the Dabao mountain tunnel mostly flows into the corresponding collapse after the surface convergence, and then flows into the underground, according to the time of each collapse of the tunnel, so $\alpha=0.82$ (integrated value).

A - catchment area, $\mathrm{m}^{2}$, the catchment area of No. 1 collapse is $30000 \mathrm{~m}^{2}$, the catchment area of No. 2 collapse is $94500 \mathrm{~m}^{2}$, the catchment area of No. 3 collapse is $15400 \mathrm{~m}^{2}$.

$\mathrm{X}$ - rainfall intensity, $\mathrm{mm} / \mathrm{h}$, according to statistics, the maximum daily precipitation is $188.2 \mathrm{~mm}$ in 20 years, is $202.5 \mathrm{~mm}$ in 30 years, is $220.5 \mathrm{~mm}$ in 50 years, and is $244.1 \mathrm{~mm}$ in 100 years in the Shaoguan area. However, on June 22, 2012, there was a rainfall intensity of $33.3 \mathrm{~mm} / \mathrm{h}$ and an extreme rainfall of $120 \mathrm{~mm} / \mathrm{h}$ on May 2, 2017.

$\mathrm{T}$ - rainfall time, $\mathrm{h}$

$\eta$ - time hysteresis coefficient, the time of appearing peak values of tracer tests are from 2 hours to 2.5 hours, so $\eta=0.6$.

Table 1. Gushing water time-process calculation $\left(\mathrm{m}^{3} / \mathrm{h}\right)$.

\begin{tabular}{lllll}
\hline rainfall intensity $(\mathbf{m m} / \mathbf{h})$ time$(\mathbf{h})$ & $\mathbf{7 . 8 4}$ & $\mathbf{8 . 4 4}$ & $\mathbf{9 . 1 8}$ & $\mathbf{1 0 . 1 7}$ \\
\hline 0.5 & 269.8 & 290.5 & 315.9 & 350.0 \\
1.0 & 539.6 & 580.9 & 631.9 & 700.0 \\
1.5 & 809.5 & 871.4 & 947.8 & 1050.0 \\
2.0 & 1079.3 & 1161.9 & 1263.7 & 1400.0 \\
2.5 & 1349.1 & 1452.3 & 1579.7 & 1750.0 \\
\hline
\end{tabular}


There are ditches with size of $35 \mathrm{~cm} * 45 \mathrm{~cm}$ on both sides of tunnel. Their maximum total drainage capacity is $1200.4 \mathrm{~m}^{3} / \mathrm{h}$. The calculation results (Table 1) show that when the rainfall intensity is $7.84 \mathrm{~mm} / \mathrm{h}$, the rainfall time that lead to the gushing water exceeds the drainage capacity of the gutter is $2.5 \mathrm{~h}$. When the rainfall intensity are $8.44 \mathrm{~mm} / \mathrm{h}, 9.18 \mathrm{~mm} / \mathrm{h}$ and $10.17 \mathrm{~mm} / \mathrm{h}$ respectively, the rainfall time that lead to the gushing water exceeds the drainage capacity of the gutter are $2.2 \mathrm{~h}, 2.0 \mathrm{~h}$ and $1.8 \mathrm{~h}$ respectively. When the rainfall intensity is $33.3 \mathrm{~mm} / \mathrm{h}$, the rainfall time that lead to the gushing water exceeds the drainage capacity of the gutter is $35 \mathrm{~min}$ only.

\section{Conclusion}

There are three karst collapses on the surface of the hill which are connected to Dabaoshan tunnel. The gushing water source of Dabaoshan tunnel is rainfall. The gushing water channels are karst collapse, karst solution fissure and fissure. When the rainfall intensity are $7.84 \mathrm{~mm} / \mathrm{h}, 8.44 \mathrm{~mm} / \mathrm{h}, 9.18$ $\mathrm{mm} / \mathrm{h}, 10.17 \mathrm{~mm} / \mathrm{h}$ respectively, the rainfall time that lead to the gushing water exceeds the drainage capacity of the gutter are $2.5 \mathrm{~h}, 2.2 \mathrm{~h}, 2.0 \mathrm{~h}, 1.8 \mathrm{~h}$ respectively. When the rainfall intensity is $33.3 \mathrm{~mm} / \mathrm{h}$, the rainfall time that lead to the gushing water exceeds the drainage capacity of the gutter is $35 \mathrm{~min}$. When a lot of water, mud and sands flow on the road, it directly impact on traffic safety, even causing traffic accidents.

\section{References}

[1] Wei Sen. Water Damage and Prevention of Railway Tunnels [J]. Teaching and Technology, 1986 (02): 30-36 (in Chinese).

[2] Wan De-you. Operation of railway tunnel water damage remediation [J]. Railway Construction, 2000 (12): 13-15 (in Chinese).

[3] Li Huai-jian. Water Remediation of a Tunnel in Bolai Expressway [J]. Railway Construction Technology, 2001 (04): 37-39 (in Chinese).

[4] Zhu He-quan, Li Haiyan. Current Status and Trends of Waterproof Technology for Railway Tunnels [J]. Railway Construction, 2003 (10): 36-38 (in Chinese).

[5] He Zhong-ming, Peng Zhen-bin, Chen Le-qiu, Wu Qi-hong. Construction Technology for Operation of Tunnel Water Damage [J]. Prospecting Science and Technology, 2005 (05): 41-43 (in Chinese).

[6] Li Xian-wei. Interaction between tunnel water damage and geological disasters and comprehensive prevention and control [D]. Xi' an Jiao tong University, 2007 (in Chinese).

[7] Xue Yu-guo, Li Shu-cai, Li Shu-zhen, Zhang Qing-song, Su Mao-xin, Zhong Shi-hang, Li Wei. Study on water hazard prediction method for high-risk tunnel construction period $[\mathrm{J}]$.
Journal of Heilongjiang Hydraulic Engineering College, 2008 (02): 117-122 (in Chinese).

[8] Xu Changchun, Wang Liang-hong, Liu Peng. Treatment of Tunnel Water Disaster Based on Chain Theory [J]. Shanxi Architecture, 2008 (13): 316-317 (in Chinese).

[9] Ma Dong. Comprehensive prevention and control technology of water disasters in complex karst tunnel of Yiwan Railway [J] China Engineering Science, 2010, 12 (08): 94-98 (in Chinese).

[10] Zhang Min. Treatment of water leakage in existing tunnels [J]. Science and Technology Information, 2011 (07): 223 (in Chinese).

[11] Huang Ke. Application of Directional Grouting Technology in Treatment of Tunnel Water Disaster-Taking Zhuzilin Station Tunnel in Shenzhen as an Example [J]. Journal of Hunan University of Arts and Science (Natural Science Edition), 2012, 24 (02): 60-63 (in Chinese).

[12] Liu Jian, Liu Dan. SVM-based source identification of tunnel water inrush [J]. Hydrogeology and Engineering Geology, 2012, 39 (05): 26-30 (in Chinese).

[13] Shi Ming. Treatment of Water Inrush and Water Leakage in Pingdong Tunnel on Banjiang Highway [J]. Science and Technology Information, 2013 (23): 369+402 (in Chinese).

[14] Zhang Yan-long, Li Qing, Tian Qing-yan. Analysis and treatment of water damage in highway tunnels operating in karst area [J]. Guangdong Highway Traffic, 2014 (04): 55-58 (in Chinese).

[15] Chen An. Water Hazard Characteristics of PiShuangAo Karst Tunnel. 2014 International Conference on Civil Engineering, Energy and Environment (ISTP). Hongkong, 2014. 12, pp 169-174.

[16] Liu Li, Liu Qun. Risk assessment of water damage in karst tunnel construction and its prevention and control measures [J]. Transportation technology, 2015 (01): 102-103 (in Chinese).

[17] Zhang Yan-long, Li Qing, Liu Hao. Study on the Mechanism and Evaluation of Water Damage of the Goupuling Tunnel in Yangjiang-Yangchun Section of Expressway [J]. Journal of Highway and Transportation Technology (Application Technology Edition), 2016, 12 (09): 193-196 (in Chinese).

[18] Li Kai, Lin Hai-shan. Evaluation of Water Damage Mechanism and Safety of Baishan Tunnel on Handan Expressway [J]. Guangdong Highway Traffic, 2017, 43 (03): $34-38$ (in Chinese).

[19] Zhang Yan-long, Liu Hao, Tian Qing-yan, Lin Hai-shan. Assessment of water damage in the Chalinding Tunnel of Guangzhao Expressway [J]. Chinese Journal of Underground Space and Engineering, 2017, 13 (S2): 860-864 (in Chinese).

[20] Zhang Yanlong, Tian Qingyan, Zhang Jiantong. Water disaster analysis and numerical simulation of a karst tunnel in a highway of Guangdong Province [J]. Carsologica Sinica, 2018, 37 (2): 307-313. 